\title{
La construcción de las realidades geográficas. Una geografía de la acción
}

Werlen, B. La construcción de las realidades geográficas. Una geografía de la acción. Santiago, Chile: Editorial Universitaria. En prensa.

( Felipe Omonte Vera

Licenciado en Sociología, Departamento de Sociología, Universidad de Chile.

Fernando Campos Medina

Profesor Asistente, Departamento de Sociología, Universidad de Chile.

La construcción de las realidades geográficas. Una geografía de la acción es una interesante compilación de escritos y textos originales del geógrafo humano Benno Werlen. Este libro es la traducción del original publicado en alemán, Gesellschaftliche Räumlichkeit Zwei. Konstruktion geographischer Wirklichkeiten, realizada por Fernando CamposMedina y Alkos Cerda, y será publicada este 2021 en Santiago de Chile, por la Editorial Universitaria. Precisamente, el objeto de esta reseña es presentar el libro de Benno Werlen como expresión principal de su trabajo académico, posicionando a la Geografía Social centrada en la acción como una perspectiva que entrega una nueva visión geográfica de las relaciones sociales, culturales, políticas, económicas y ecológicas.

En el Capítulo 1, Constitución de relaciones espaciales, el autor presenta su perspectiva discutiendo la relación entre espacio y sociedad. Esta discusión nos lleva a las reflexiones que se establecieron en la constitución de la geografía como disciplina científica, revisitando a grandes pensadores occidentales como Newton, Leibniz y Kant. El motivo de este ejercicio reflexivo resulta fundamental para abordar el estudio de las condiciones espaciales y temporales actuales, así como también para la propuesta de Werlen de una geografía sin espacio. Desde este planteo, el autor distinguirá entre dos tipos de geografía: una tradicional y una orientada a la acción de los individuos.

La geografía tradicional forma parte de lo que Werlen denomina ciencia del espacio, cuya tradición se remonta al esquema territorialista propuesto por uno de los pioneros de la geografía moderna: Alfred Hettner. Aquí, el énfasis está en reconocer al espacio, en cuanto espacio terrestre, como determinante y causante de las relaciones sociales que se gestan en una sociedad. Así, este se vincula a una noción de contenedor de la realidad social, dotado de una eficacia causal y un potencial determinador de las actividades de los sujetos; una perspectiva en que la investigación geográfica opera bajo el supuesto de anclamiento, en el cual las rutinas de acción y las tradiciones se delimitan espaciotemporalmente. Para el autor, la preponderancia de este punto de vista en la investigación geográfica radica en las características de las sociedades tradicionales que -a primera vista- hacían pertinente el esquema territorialista de Hettner. No obstante, dicho modelo ni siquiera es apropiado para las sociedades tradicionales, por la inviabilidad de observar las realidades sociales, culturales y económicas en una superficie terrestre particular: solo los artefactos materiales pueden ser localizados y regionalizados, pero no los valores culturales y normas sociales que se encuentran en ellos.

La llegada de las sociedades moderno-tardías refleja la poca solvencia de la ciencia del espacio en la investigación geográfica. Las tradiciones pierden influencia en los individuos y ya no trascienden cada aspecto de sus vidas. Las decisiones individuales tienen un marco más amplio, posibilitando una mayor conducción de las distintas esferas de su vida. En las relaciones sociales, las actividades económicas se posicionan por sobre las relaciones de parentesco. En este sentido, las relaciones en la sociedad se explicarían más por el rol dentro de los procesos de producción, que por la edad 
y, teóricamente, por el género. Situación que sin duda ha empezado a cambiar en las últimas dos décadas.

En ese contexto, Werlen enfatiza que los territorios, o el espacio per se, no pueden ser el objeto de estudio de la Geografía Social, sino que la investigación debe centrarse en las actividades humanas bajo condiciones espaciales específicas. Es necesario abandonar la idea de investigar la superficie terrestre para adentrarse en las geografías creadas y reproducidas cotidianamente por los actores, según sus diferentes posiciones de poder. Por lo tanto, el concepto de espacio debe entenderse como una conceptualización de la realidad físicomaterial que sea relevante para la corporalidad de los sujetos. El autor lo define como un concepto clasificatorio formal, contrario a la noción empírica o apriorista con la que se lo solía delimitar desde otras perspectivas. Siendo clasificatorio por hacer posibles descripciones que ordenan los objetos materiales y la orientación del mundo físico; y formal por no apuntar a características relacionadas con el contenido de elementos materiales.

El segundo Capítulo, El giro teórico cultural, propone que cultura y política deben pensarse de manera conjunta, producto de la influencia del cultural turn en la perspectiva geográfica. Aquí se profundiza en el debate sobre identidad desde la geografía, centrándose, en palabras de Werlen, en la pregunta acerca de quién y con qué puede proveerse de un tipo de identidad y de qué forma pueden ser usadas las preguntas sobre identidad en la perspectiva geográfica.

Siguiendo esta línea de pensamiento, en su análisis sobre identidad cultural, el autor presenta dos posturas opuestas (estructuralistas/funcionalistas e individualismo metodológico) en cuanto a la comprensión de la cultura, mostrando las falencias de ambas. Werlen critica las posiciones que definen a la cultura como un ente que ejerce presión determinante en los individuos (posturas estructuralistas/ funcionalistas) y aquellas donde la cultura remite solamente a la individualidad de los sujetos (individualismo metodológico). A modo de respuesta, se propone como tercera vía, una línea argumentativa ligada a la Teoría de la Estructuración de Anthony
Giddens. En ella se aceptan conceptos como estructuración (la reproducción de las prácticas en donde las estructuras se forman) y dualidad de la estructura (las estructuras sociales están constituidas por la actividad humana y, a la vez, son el medio de esta constitución) para construir una definición de cultura coherente con la Geografía Social.

Una de las contribuciones de la teoría de Giddens se encuentra en compatibilizar el individualismo metodológico con la dualidad de la estructura, conectando los niveles micro y macro del análisis. Es decir, la variante institucional del individualismo remarca el significado liberador y restrictivo de las instituciones. La Geografía Social incluye al individualismo metodológico como concepto básico, tanto en el marco de las perspectivas objetivas (énfasis en la transformación o reproducción de las estructuras como tales) como de las subjetivas (enfoque en los objetivos y motivos de la acción, así como en sus consecuencias para la estructuración). De esta forma, se propone un concepto de cultura que no puede entenderse como objeto de investigación sui generis, sino como categoría analítica o como dimensión y resultado de la acción. Se define la cultura como el resultado de acciones pasadas y requisito para el comportamiento futuro de los individuos. Esto involucra no solo productos materiales, sino también sistemas de valores o reglas.

A partir de ese concepto es posible preguntarse por la identidad, afirmando que en los espacios de vida es imposible atribuir identidad per se, porque lo cultural y lo social no pueden ser reducidos a la superficie terrestre. Los significados culturales de la acción se relacionan con el contexto de la acción y sus formas de representación. Uno de los criterios objetivos de la identidad local, entonces, se encuentra a nivel de las representaciones sociales, especialmente, en el trabajo simbólico, donde los líderes buscan que las personas a las que representan acepten sus propios intereses. Así, en lugar de estudiar identidades regionales, es mejor preguntarse por los aspectos regionales y las condiciones de la identidad cultural. Como guía de análisis, Werlen se basa en la Teoría de las Formas de Vida de Alfred Schutz en pos de comprender el origen de los patrones de interpretación compartidos entre 
individuos. Para constituir un sentido intersubjetivo, se requiere de una comprobación inmediata de las atribuciones de sentido y de que las experiencias compartidas por los individuos sean importantes para esa identidad. En ese proceso, la copresencia (face-to-face) es clave en la comprobación directa de los contenidos de comunicación. Más aún en un contexto donde la globalización ha establecido un desanclamiento espacial y temporal, en el que ambos componentes ya no están conectados de manera fija.

El Capítulo 3, Territorialización y globalización, como sugiere su nombre, el autor se enfoca en dos conceptos: territorialización y globalización. Durante una entrevista, Werlen muestra la importancia de Google Earth que, junto con los nuevos medios de información, es expresión de un rasgo característico de las sociedades modernas: la capacidad de poder actuar y comunicar a través de largas distancias. El individuo puede integrar su propio contexto de vida dentro de un contexto global, en una era que elimina la diferencia temporal entre transporte e información. Desde un punto de vista filosófico, también se puede observar este desarrollo. Para la Ilustración, el entendimiento de uno mismo incluye comprender dónde estoy y dónde me encuentro. Sobre estos principios se vuelve posible ordenar la vida propia en nuevos contextos de significado, al mismo tiempo que el desarrollo específicamente nacional o regional va perdiendo su influencia.

Ahora bien, este proceso de transformación presenta una contradicción ya que, si bien las posibilidades de comunicación suprimen las barreras espaciales, la política nacional-estatal y el derecho siguen anclados territorialmente. Esto pone en el debate a los regionalismos y nacionalismos, que comienzan a tener mayor importancia. La globalización debe ser vista como un fenómeno dialéctico, porque precisamente la expansión de la interdependencia global es lo que ha llevado a un auge del nacionalismo y la identidad local. Estas posturas se basan en categorías territoriales que potencian el sentimiento de pertenencia hacia el interior, diferenciándose del exterior en sentido positivo o negativo. Como discurso político, ambas tendencias utilizan un componente fundador de identidad para lograr sus objetivos. Así, se establecen y reproducen mediante la carga simbólica de lugares y determinados artefactos materiales. Esta ontologización del espacio se relaciona directamente con la retórica de sangre y suelo de los nacionalismos (o regionalismos) demagógicos.

Con todo, la tarea de la Geografía Social se encuentra en abandonar la atención que por siglos se ha mantenido sobre los territorios nacionales o regionales. Así, las regiones deben considerarse espacios de experiencia humana que promueven la copresencia, a modo de desarrollar y mantener la identidad local y social en el medio urbano y rural. A lo largo de este proceso, es importante el rol que juega la ciencia y las tipificaciones que se hacen sobre las relaciones sociales generadas en un territorio específico. Werlen observa en la historia de Europa el fiel reflejo de que, en contextos de diversidad cultural donde previamente existieron comunidades locales, las visiones relacionadas con la biología y la pertenencia nacional tienen resultados devastadores. Tanto la ciencia como las naciones deben y pueden existir sin nacionalismos demagógicos, permitiendo hacer justicia al carácter social y constructivo de la regionalización. Para el autor, esta es la responsabilidad política de los/as geógrafos/as contemporáneos y futuros.

El Capítulo 4, Prácticas sociales y la geografía de la vida propia, es una profundización de los contenidos propuestos en el capítulo anterior, especialmente, los relacionados con el concepto de regionalización cotidiana. El autor presta atención a las prácticas sociales como base de una visión dinámica del mundo geográfico. Se vuelve a recalcar que las condiciones espaciales son portadoras de sentido, las cuales se generan por la atribución que brindan los propios actuantes. Su vinculación al mundo toma importancia al ser resultado del control por parte de los individuos de las relaciones sociales espaciales de la acción, principalmente en aquellas de expansión supralocal y global.

Este capítulo comienza con una cita de Kant, en la cual se establece que el sentido fundamental de la geografía es su capacidad de iluminar el entendimiento humano. Así, por medio de la descripción de la superficie terrestre, las personas pueden posicionarse en un contexto espacial determinado y tener 
una orientación ilustrada. Por lo tanto, el conocimiento geográfico es un requisito central para el establecimiento de la modernidad y sus formas de vida. Actualmente, las condiciones geográficas son fundamentales en la ejecución de las actividades humanas como nunca antes se había presenciado. En tiempos globalizados, las condiciones geográficas fundamentales ya no se limitan a saber el lugar dónde se presentan las cosas, sino que se encuentran en la geografía de los sujetos.

La importancia de la geografía de los sujetos se encuentra en que ella brinda las mismas posibilidades que la globalización, es decir, permite comprender que las personas desarrollan su vida diaria en un entorno local, al mismo tiempo que sus estilos de vida se encuentran inmersos en procesos globales. Sobre esta base, la constitución de cada realidad descansa en un tipo específico de quehacer geográfico cotidiano. Su investigación se dirige al desarrollo de una conciencia geográfica acorde a los tiempos actuales, reconfigurando la relación entre sociedad y espacio que se tenía en la geografía tradicional. Se genera, así, una ciencia basada en una concepción del mundo enfocada en la experiencia cotidiana de la globalización (Werlen la denomina experenciabilidad de la globalización en lo cotidiano).

El quinto Capítulo, Ecología Social, es el intento de Werlen por abarcar, desde su perspectiva, la relación entre sociedad y naturaleza. Para él, la Geografía Social se debe preocupar por tener un carácter ecológico, desarrollando una Ecología Social sin reduccionismos entre sociedad y naturaleza. Por esta razón, el autor profundiza en discusiones ontológicas y metodológicas relacionadas con problemas ecológicos, afirmando que no sólo se debe abogar por un cambio disciplinar, sino que también es necesario intervenir en la realidad social que se investiga.

Ahora bien, para analizar los fenómenos relacionados con la ecología, los geógrafos requieren de un trabajo multidisciplinar (integrativo). Es necesario resolver y darse cuenta de las falencias que puedan existir en la Geografía Social y entender que las ciencias sociales y las ciencias naturales por sí solas no pueden aclarar el vínculo entre componentes socioculturales y las situaciones ecológicas problemáticas. Para Werlen, la solución está en integrar, de manera adecuada, el recorrido teórico que existe en la Teoría de la Acción impulsada por las ciencias sociales.

A lo largo del capítulo, es posible apreciar que el trabajo de investigación teórica y empírica de una Ecología Social se orienta al potencial tecnológico y político de transformar la naturaleza, tomando en cuenta los componentes del poder que se encuentran en esas dimensiones. Las sugerencias de reestructuración de espacios, paisajes, unidades ecológicas, relaciones sociales con la naturaleza, etc., solo pueden implementarse junto con las posiciones de poder social de los individuos. Por lo tanto, la investigación no puede remitirse a algo externo a los individuos, en cuanto supone una justificación de sus acciones. Así, la propuesta centra su atención en los motivos de dicha acción y los medios por los cuales ciertos individuos aseguran su poder, como las causas directas de la crisis ambiental que se vive en la actualidad.

El manuscrito culmina con un epílogo que invita a reflexionar sobre el futuro del comportamiento geográfico, en un contexto donde las relaciones sociales espaciales se manifiestan de una manera nunca antes vista. El llamado a un cambio disciplinar es urgente, ya que la concepción de mundo que presenta la geografía tradicional no es coherente con las condiciones geográficas cotidianas de la acción. Para Werlen, es necesario insistir en un acoplamiento entre la ciencia y lo cotidiano, particularmente, en tiempos donde las lógicas territoriales siguen teniendo influencia en la investigación geográfica. Además del programa formulado, se incluye la exploración sistemática de las relaciones espaciales vinculadas al mundo y su significado para la realidad sociocultural, sustituyendo la lógica de los espacios (lugares) por una lógica geográfica-social de las prácticas. Sin embargo, vale destacar el potencial conflicto que observa Benno Werlen entre la transformación de las relaciones espaciales sociales a escala global y el hecho de que los Estados nacionales sigan resolviendo las problemáticas de la globalización bajo estrategias tradicionales (territoriales). Por ello, junto a un nuevo lenguaje de lo espacial, es necesaria una 
La construcción de las realidades geográficas. Una geografía de la acción

comprensión renovada de las relaciones sociales, tanto en el marco de nuevas potencialidades de la acción a distancia como de la aceleración de los procesos sociales vinculados.

Felipe Omonte Vera / felipe.omonte@ug.uchile.cl Licenciado en Sociología, Universidad de Chile. Estudiante del Magíster en Gestión y Políticas Públicas, Facultad de Ciencias Físicas y Matemáticas, Universidad de Chile. Investigador Laboratorio de Sociología Territorial (LST). Sus temas de investigación se vinculan a temáticas de discapacidad y estudios territoriales con un enfoque de redes de actores.
Fernando Campos Medina / fernandocampos@uchile.cl Sociólogo de la Pontificia Universidad Católica de Chile. Máster en Vivienda y Urbanismo, Universidad Politécnica de Cataluña. Doctor en Sociología, Universidad Friedrich Schiller de Jena, Alemania. Doctor en Urbanismo, Bauhaus Universidad de Weimar, Alemania. Director Laboratorio de Sociología Territorial (LST). Se desempeña como profesor asistente del Departamento de Sociología de la Universidad de Chile. Sus temas de investigación son Teoría del Territorio, Sociología Ambiental, Sociología Urbana y Políticas Públicas Territoriales, siendo investigador responsable de múltiples proyectos asociados a estas temáticas. 